\title{
El interaccionismo simbólico: oportunidades de investigación en el aula de clase
}

Symbolic Interactionism: Research Opportunities in the Classroom

\author{
Juan Carlos Piñeros Suárez ${ }^{1}$ \\ Secretaría de educación de Bogotá
}

Recibido: 13.12 .2020

Aceptado: 27.12.2020

\section{Resumen}

El presente artículo de reflexión expone algunas de las situaciones particulares que se presentan en el aula de clase desde la triada enseñanza, saber y aprendizaje. Caracteriza algunas dificultades que se han abordado históricamente en la estructuración del saber pedagógico, sus incidencias en la visión presente de la educación y las afectaciones a la labor docente. Posteriormente se propone un abordaje desde el interaccionismo simbólico como un presupuesto epistémico que puede aportar en la investigación educativa y a su vez, sir ve de apoyo en el mejoramiento de las reflexiones que realiza el docente en el entorno escolar. Para finalizar, se profundiza en la teoría fundamentada como un diseño metodológico que puede ayudar en la sistematización de las experiencias del aula de clase y el desarrollo de los proyectos de investigación en la escuela.

Palabras clave: Interaccionismo simbólico, Investigación escolar, Teoría fundamentada.

\footnotetext{
1 jcpineros@educacionbogota.edu.co https://orcid.org/0000-0001-9905-9317

https://scholar.google.es/citations?user=DVnEwo4AAAAJ\&hl=es
} 


\begin{abstract}
This reflective article exposes some of the particular situations that emerge in the classroom from the triad of teaching, knowing and learning. It characterizes some difficulties that have historically been addressed in the structuring of pedagogical knowledge, its effects on the current view of education and the effects on teaching. Subsequently, an approach from symbolic interactionism is proposed as an epistemic assumption that can contribute to educational research and, it also supports in the improvement of the teacher reflections in the school environment. Finally, the grounded theory is studied deeply as a methodological design that can help in the systematization of classroom experiences and the development of research projects at school.
\end{abstract}

Keywords: Symbolic interactionism, School research, Grounded theory.

\title{
1. Contexto
}

Las prácticas de enseñanza, saber y aprendizaje evidencian un estancamiento en relación a los cambios que se presentan en la sociedad a nivel personal, tecnológico, social, cultural y educativo. Se vivencia un momento en que los educadores deben ser gestores de conocimiento, mediadores entre el estudiante y las competencias del siglo XXI, conocedores de las realidades personales de sus educandos e innovadores en los campos disciplinares en los cuales se desempeñan. Estas competencias, que son necesarias en los educadores como una posibilidad de adaptarse a las trasformaciones que exige un mundo globalizado, deben ser fortalecidas, potenciadas, estimuladas y desarrolladas desde el ámbito educativo en la formación de futuros docentes y en la vivencia profesional diaria. Al respecto (Gardner y Davis, 2014) son claros al afirmar que la educación del presente nos debe preparar para un futuro donde los jovenes están bastante permeados por nuevas formas de entender las realidades y los tutores no pueden mantener una postura rigida frente a estos cambios. 
Es notable que en la escuela de los contextos menos favorecidos hay problemáticas afectan a los estudiantes y obligan a los educadores a fortalecer campos del conocimiento que no necesariamente pertenecen a sus áreas de desempeño. La ausencia de un núcleo familiar compuesto, los problemas de pandillas y drogadicción, las escasas oportunidades de una educación de calidad, los altos índices de embarazo, la falta de motivación en la asistencia a clases, los modelos pedagógicos rígidos y las políticas públicas que no encuentran asertividad en medio de las necesidades colectivas, son factores que deben incidir en la perspectiva que tiene el educador en estos contextos, ya que

La adolescencia es también el momento en que deben consolidarse los hábitos de aprendizaje para el resto de la vida. La adquisición de la disciplina, el hábito de trabajo intenso y constante, debe comenzar mucho antes. Pero es durante la adolescencia cuando la postura individual con respecto a las verdades y a la manera en que se seguirán estableciendo cuando concluya la escolarización formal se consolida como un hábito mental para toda la vida. (Gardner, 2011, p. 180)

Considerando estos fundamentos, el educador se posibilita como un agente de transformación y cambio, siempre que reconozca las fortalezas, debilidades y posibilidades de gestión en el contexto donde se desenvuelve. No obstante, en Colombia, el docente ha disminuido ese rol de investigador y productor de conocimiento, por diferentes factores que han afectado las posibilidades de influir positivamente en la sociedad desde su saber pedagógico. Al respecto, la investigación historia de las practicas pedagógicas en Colombia descrita por (Zuluaga, 2003) nos indica que en la década de los años 70 y 80 el docente en el país estuvo supeditado a lo que la estructura curricular prescrita por el ministerio de educación le ofertaba desde los contenidos y métodos que deberían ser utilizados como única opción para enseñar al colectivo educativo. Esta mirada de lo educativo, termino involucrando en las escuelas algunas disciplinas del conocimiento, como la sociología de la educación, la administración educativa, la psicología de la educación, entre otras, lo cual, afecto notablemente la capacidad reflexiva del maestro y su posibilidad de generar rigurosidad en los procesos de enseñanza, puesto que, cada decisión tomada en la escuela estaba mediada por otras ramas del saber con mayor prestigio académico. 
Al desdibujar este rol educativo, el docente se acostumbra a la administración del currículo, dejando de lado el saber pedagógico, la investigación en el aula, el crecimiento intelectual y la reflexión propia de su labor pedagógica. Esta problemática histórica invita a los educadores a generar reflexiones profundas sobre la educación, la pedagogía, el saber didáctico, las nuevas realidades sociales mediadas por las tecnologías emergentes, las problemáticas derivadas de las situaciones sociales antes descritas, los retos y desafíos del siglo XXI como una oportunidad de describir, interpretar y conocer sus contextos de trabajo, su población educativa, la variedad de aprendizajes necesarios para la vida y la multiplicidad de formas de aprender que tienen los jóvenes en esta generación. En este sentido, se propone una reflexión desde el aspecto investigativo como una posibilidad para evaluar los procesos de avance a nivel pedagógico desde el quehacer educativo del docente en las aulas de clase. Se acude al interaccionismo simbólico como un presupuesto epistémico que permite al docente evaluar las relaciones que se presentan dentro y fuera del entorno escolar, como un insumo para estructurar unidades didácticas acorde a las realidades personales, sociales y culturales que demanda el momento presente.

\section{El interaccionismo simbólico}

El interaccionismo simbólico se concibe como un presupuesto epistémico abordado desde el pragmatismo, es utilizado en procesos investigativos en el campo de la sociología, la psicología y hace unos pocos años en el campo de la educación. "Entre los numerosos especialistas que han utilizado dicho enfoque o contribuido a su consolidación intelectual, figuran autores norteamericanos tan notables como: George Herbert Mead, Jhon Dewey, W.I. Thomas, Robert E. Park, William James, Charles Horton Cooley, Florian Znaniecki, James Mark Balwin, Robert Redfield y LouisWirth” (Blumer, 1982, p. 1). Sus inicios se remontan a mediados del siglo XX en la escuela de chicago, específicamente en el campo de la sociología, donde rápidamente incursiona en la psicología y otras ramas del saber cómo la antropología. El interaccionismo simbólico tiene tres características que permiten identificar su posición en el ámbito de la investigación, la primera está relacionada con el direccionamiento de los actos de la persona en función del significado de estos actos, la segunda característica es que los 
actos y el significado de los mismos se relacionan con la interacción social y la tercera característica es que los significados son manipulables y modificables a través de la interpretación que hace la persona al exponerse a las situaciones cotidianas (Blumer, 1982).

Desde esta perspectiva, el significado se propone como un efecto secundario de la interacción, por lo cual los sujetos comprenden el mundo a través de la confrontación de realidades que confluyen en una modificación propia de la existencia, considerando que las diferentes culturas desarrollan procesos de aprendizaje que están ligados a las realidades particularidades del contexto, las costumbres o hábitos heredados y la perspectiva de sociedad que se imagina construir. Estos factores inciden en la manera en la que se comprende el mundo, al considerar que cada persona estructura una visión o un imaginario de la realidad mediado por el núcleo social en el cual se forma. Por lo tanto, las realidades construidas varían según las relaciones que se estructuran al compartir y convivir con los otros, al respecto, Piaget nos indica que "salvo las dificultades del lenguaje finalista, la adaptación debe caracterizarse como un equilibrio entre las acciones del organismo sobre el medio y las acciones inversas" (Piaget, 1999, p. 17).

Considerar a la interacción social como base del comportamiento de las estructuras sociales, implica tener en cuenta a la persona que consolida las relaciones y brinda sentido desde su interpretación simbólica o no símbólica de las realidades existentes, la persona desde una caracterización intrínseca o la persona que emerge en comunidad, en palabras de (Mounier, 1974)

una persona es un ser espiritual constituido como tal por una forma de subsistencia y de independencia en sus ser; mantiene esta subsistencia mediante su adhesión a una jerarquía de valores libremente adoptados, asimilados y vividos en un compromiso responsable y en una constante conversión; unifica así toda su actividad en la libertad y desarrolla por añadidura, a impulsos de actos creadores, la singularidad de su vocación. (p. 603) 
Esta mirada del aspecto personal no supone en ningún momento un avance hacia el individualismo, por el contrario, se desprende del ego absoluto para observar en los otros "más allá de su objetivación actual, supraconsciente y supratemporal, más amplia que las visiones que de ella tengo, más interior que las construcciones que de ella intento" (Mounier, 1974, p. 609). Este punto de vista, explica de forma clara algunas de las características del interaccionismo simbólico, la necesidad de un mensaje comprendido por las dos partes basado en la apropiación de un símbolo que se ha identificado y se le ha dado un significado, la identificación de objetos en el contexto que son representados para los sujetos y que supone un conocimiento en común para el colectivo social y la naturaleza de las acciones que llevan a las personas a actuar de una forma $u$ otra.

\section{Metodología e investigación en el interaccionismo simbólico}

Para decantar los aspectos metodológicos del interaccionismo simbólico debemos hacer una reflexión sobre la emergencia de la perspectiva interpretativa como una posibilidad para generar cientificidad en los fenómenos e interacciones sociales, teniendo en cuenta que las investigaciones de carácter social buscan posibilitar interpretación y descripción de las particularidades en un contexto determinado y las investigaciones de corte cuantitativo intentan explicar desde el método científico tradicional resultados medibles o predecibles con variables predeterminadas. Básicamente para comprender la perspectiva metodológica abordada en este escrito debemos partir de que,

El comportamiento humano está principalmente constituido por acciones, siendo característica definitoria de las mismas poseer un sentido para quienes las realizan y en convertirse en inteligibles sólo en la medida en que se conoce el sentido que le atribuye el actor individual. Es por ese motivo que las acciones sociales no pueden observarse del mismo modo que los procesos naturales. (Esteban, 2003, p. 58)

Por lo tanto, la hermenéutica, la fenomenología y el interaccionismo simbólico pretenden interpretar y comprender el comportamiento humano desde las realidades del sujeto que 
interactúa, las características del investigador, las fuentes teóricas sólidas, la emergencia de los datos y su categorización rigurosa "in situ". En este orden de ideas, el interaccionismo simbólico se considera "como una perspectiva dentro de la ciencia social empírica, un enfoque encaminado a ofrecer un reconocimiento verificable de la vida de grupo y el comportamiento humano" (Blumer, 1982, p. 16). En este propósito propende por una investigación de corte naturalista que se basa en la exploración y la inspección a traves de las realidades que emergen en la interacción,

Consecuentemente, la postura metodológica del interaccionismo simbólico es la del examen directo del mundo empírico social. Esta doctrina sostiene que el examen directo permite al especialista satisfacer todos los requisitos básicos de la ciencia empírica: enfrentarse a un mundo empírico suceptible de observación y análisis, sucitar problemas abstractos con respecto al mismo, reunir los datos necesarios a través de un examen detenido y disciplinado, descubrir las relaciones entre las respectivas categorías de estos datos, formular proposiciones a un esquema teorético y verificar los problemas, datos, relaciones, proposiciones y teoría por medio de un nuevo examen del mundo empírico. El interaccionismo no sucumbe a la mítica creencia de que un estudio, para ser cientifico, tiene que configurarse de forma que se acomode a un protocolo preestablecido de investigación cientifica. (Blumer, 1982, p. 35)

Por lo tanto, como perspectiva metodológica encuentra el rigor investigativo en el tratamiento de la información, la multiplicidad de las interacciones, la confrontación teórica en cada una de las etapas del proceso y el examen directo del mundo social empírico desentrañado y evidenciado de forma rigurosa. Para cumplir con este objetivo acude entre otros diseños metodológicos, a la teoría fundamentada, como una oportunidad de sistematizar de forma ordenada los principales hallazgos en el proceso investigativo. 


\section{La teoría fundamentada}

La teoría fundamentada se concibe como un diseño metodológico cuya principal intención es comprender la realidad emergente a partir de la recolección, interpretación y tratamiento riguroso de los datos. "Debido a que las teorías fundamentadas se basan en los datos, es más posible que generen conocimientos, aumenten la comprensión y proporcionen una guía significativa para la acción” (Strauss y Corbin, 2002, p. 22). A nivel educativo la teoría fundamentada puede ser puesta en práctica en el aula de clases a partir de la evaluación de aspectos relevantes en el quehacer pedagógico, tales como la metodología de enseñanza, la didáctica, las necesidades en los aprendizajes, las innovaciones pedagógicas y el fortalecimiento de competencias para la vida a través del proceso formativo. En este sentido, la teoría fundamentada proporciona una secuencia metodológica para comprender e interpretar el mundo escolar y sus interacciones a partir del tratamiento de la información que proveen las percepciones de los diferentes actores educativos. Desde la tradición interaccionista la teoría fundamentada emerge según Strauss y Corbin (2002), por

a) la necesidad de salir al campo para descubrir lo que sucede en realidad; b) la importancia de la teoría, fundamentada en los datos, para el desarrollo de una disciplina y como base para la acción social; c) la complejidad y variabilidad de los fenómenos de la acción humana; d) la creencia de que las personas son actores que adoptan un papel activo al responder a situaciones problemáticas; e) la idea de que las personas actúan con una intención; f) la creencia de que la intención se define y redefine por la interacción; g) una sensibilidad a la naturaleza evolutiva y en desarrollo permanente de los acontecimientos (procesos) y h) la constancia de la relación entre las condiciones (la estructura), la acción (el proceso) y las consecuencias. (p. 18)

Estas características proveen a este diseño metodológico unas posibilidades amplias para extraer de forma rigurosa las realidades que se ocultan en la interacción escolar, indagando por la percepción que se tiene de los aspectos simbólicos con los que se relaciona la cultura 
en los entornos educativos. El docente que desarrolle este trabajo desde una apuesta epistemológica basada en el interaccionismo simbólico y con un diseño desde la teoría fundamentada debe considerar algunos elementos metodológicos propuestos por (Strauss y Corbin, 2002).

\section{Formular la pregunta de investigación y determinar la problemática}

En cada contexto educativo se convive con realidades específicas que invitan al educador a conocer, comprender e interpretar las situaciones que se van presentando en relación con el clima institucional, el aprendizaje de los estudiantes, las metodologías de enseñanza, los aspectos externos que inciden en la motivación de los jóvenes y las consecuencias de estos factores en el éxito de una sociedad. Estas observaciones que realiza el docente usualmente se materializan en cuestionamientos, dudas y posibilidades de intervención, que finalmente en el campo investigativo se clasifican como interrogantes guías o problemas contextuales, al respecto Ramírez (2010) afirma que las preguntas de investigación y las situaciones problémicas surgen como,

producto de la observación cotidiana, la necesidad de hacer diagnósticos para develar las causas de algún problema, ser producto de investigaciones que sobre un tema se estén realizando y que generan problemas colaterales de investigación, por indagaciones exhaustivas de una problemática en particular y que obliga a profundizar aún más sobre la misma área, producto de la reflexión sobre diversas áreas de la realidad poco estudiadas, por la reflexión sobre aspectos aparentemente intrascendentes de la realidad social. (p. 8)

Por esto es necesario, al cuestionarse sobre una problemática de investigación considerar el tiempo con el que se cuenta para llegar a buen término, los antecedentes a nivel internacional, nacional y local sobre el tema investigado, las sub- problemáticas o componentes estructurales del problema, la unidad de análisis y las posibilidades de evaluarla y las limitaciones que se pueden presentar y el interés del investigador por la problemática a desarrollar. 


\section{El uso de los textos de apoyo y los antecedentes de la temática a investigar}

El docente desde su historia académica trae consigo una serie de teorías, enfoques, métodos y documentos propios de su saber disciplinar, el uso que dé a cada texto conocido depende en gran medida de su mirada sobre el plano investigativo y sobre la perspectiva que tiene de la educación. Es necesario, para estructurar una teoría fundamentada, que los apoyos teóricos o antecedentes de investigación representen un soporte sólido que pueda dialogar con la perspectiva del investigador, la realidad social investigada y la interpretación de los datos emergentes. Es por esto que se considera la propuesta de (Strauss \& Corbin, 2002) en relación con el correcto uso de la literatura técnica en este proceso investigativo, en primer lugar, los autores consideran ideal comparar los conceptos emergentes cuando son muy similares a la teoría, desde sus propiedades y dimensiones para diferenciar y dar características específicas a los términos emergentes. Como segunda recomendación es necesario distanciarse de los conceptos emergentes de una manera objetiva, siempre cuestionándose si es un término que surge de la investigación o tengo afinidad desde mi historia investigativa con este nuevo concepto. En tercer lugar, "Cualquier tema o concepto prestado de otro estudio puede ser de importancia para el problema investigado. Sin embargo, el investigador ha de tener mucho cuidado en buscar ejemplos de incidentes en sus datos y delinear las formas que los conceptos adoptan en el presente estudio" (Strauss \& Corbin, 2002, p. 56). Como cuarta recomendación es útil siempre estar situado desde un presupuesto epistémico para brindar propiedades a los datos desde una línea de trabajo filosófico. En quinto lugar es ideal utilizar las fuentes bibliográficas como un aporte a la estructuración de fundamento, ya que otras investigaciones traen un aservoi metodológico que puede ser útil a la investigación en desarrollo. Como sexta recomendación, es indispensable acudir a las investigaciones relacionadas con el tema antes de formular nuestra ruta metodológica, para desarrollar un camino que represente menos complejidad y prosibilite resultados con mayor coherencia. Por último, "Traer la literatura a la escritura no solamente demuestra erudición, sino que permite extender, validar y refinar el conocimiento del área. Lo que el investigador debe evitar es sentirse inseguro con sus descubrimientos. Salir corriendo en busca de la literatura publicada para validar o negar todo 
lo que uno encuentra, impide el progreso y ahoga la creatividad." (Strauss \& Corbin, 2002, p. 58)

\section{Tratamiento de la información}

La recolección de los datos en la teoría fundamentada y su aplicación al campo educativo puede darse utilizando algunas técnicas como son, los cuestionarios, la observación y la entrevista, entre otros. Sin embargo, el tratamiento de los datos sin importar la técnica de recolección de la información desde la perspectiva de (Strauss \& Corbin, 2002), se realiza mediante un proceso de codificación abierta, codificación axial, codificación selectiva y muestreo teórico. La codificación es el proceso en el cual se "condensan el grueso de nuestros datos en unidades analizables, creando categorías con ellos o a partir de ellos" (Coffey y Atkinson, 1996, p. 31). En palabras claras "La codificación es el modo en que usted define de qué tratan los datos que está analizando. Implica identificar y registrar uno o más pasajes de texto u otros datos como parte de cuadros que, en cierto sentido, ejemplifican la misma idea teórica o descriptiva" (Hibbs, 2012, p. 76). Teniendo como base estos conceptos, a continuación, se va a explicar en detalle los cuatro momentos de la codificación en la teoría fundamentada, para brindar al docente investigador una mirada clara de este proceso.

\section{La codificación abierta}

Este proceso en el tratamiento de los datos consiste en la identificación de los principales conceptos, otorgando a los datos recolectados propiedades y dimensiones. "Durante la codificación abierta, los datos se descomponen en partes discretas, se examinan minuciosamente y se comparan en busca de similitudes y diferencias" (Strauss \& Corbin, 2002, p. 111). Cuando se encuentran similitudes o particularidades que los agrupan en un escenario similar se denominan categorías y subcategorías, las cuales emergen de conceptos que posteriormente han sido clasificados y a los cuales se le ha brindado un significado. Al caracterizar estos datos desde sus propiedades y dimensiones se puede generar unas categorías más amplias, por ejemplo, si se encuentran que los jóvenes en el ámbito universitario prefieren 
asistir a clases con sus compañeros por el compartir, el debate o las diferencias en los puntos de vista, estos tres factores se pueden codificar en una categoría llamada interacción social.

\section{La codificación axial}

Esta codificación se desarrolla cuando hay correlaciones entre las categorías y las subcategorías en palabras de Strauss \& Corbin (2002) "El propósito de la codificación axial es comenzar el proceso de reagrupar los datos que se fracturaron durante la codificación abierta. En la codificación axial, las categorías se relacionan con sus subcategorías para formar unas explicaciones más precisas y completas sobre los fenómenos” (p. 135). En este aspecto es necesario conceptualizar y caracterizar cada una de las categorías y subcategorías desde su significado y el concepto que emerge desde la interpretación por parte del investigador en correlación con el presupuesto epistémico o paradigma de investigación, estructurando una visión amplia que permita comprender las causalidades que dan forma al fenómeno estudiado.

\section{Codificación selectiva}

En este proceso se hace un tratamiento especial a los datos con el objetivo de ser refinados, intentado considerar las partes como un todo, al respecto Strauss y Corbin (2002) plantean:

En la codificación abierta, el analista se preocupa por generar categorías y sus propiedades, y luego busca determinar cómo varían en su rango dimensional. En la codificación axial, las categorías se construyen de manera sistemática y se ligan a las subcategorías. No obstante, sólo cuando las categorías principales se integran finalmente para formar un esquema teórico mayor, los hallazgos de la investigación adquieren la forma de teoría. La codificación selectiva es el proceso de integrar y refinar las categorías. (p. 157)

En la codificación selectiva el conocimiento, la experticia en el manejo de los datos y la capacidad de leer las categorías, subcategorías, fenómenos y realidades, brinda un papel 
relevante al investigador que tiene la función de estructurar un andamiaje teórico sólido, explicado desde la emergencia de los datos y confrontado con la teoría existente, a este proceso se le denomina integración. En la integración el investigador encuentra la categoría central, la cual tiene relación con cada momento del proceso y permite refinar la teoría desde la coherencia y la cohesión para llegar a una validez teórica sustentada desde cada una de las partes.

\section{Muestreo teórico}

Como los expone (Flick, 2012) el muestreo se divide en estadístico, el cual es muy utilizado en las ciencias experimentales y propone la muestra a evaluar desde el planteamiento inicial de la investigación y en muestreo teórico el cual está relacionado con la unidad de análisis que se va seleccionando en el proceso, siempre en relación con la codificación abierta, axial y selectiva. En este escenario la muestra a evaluar no se conoce previamente, por el contrario, a medida que se avanza en cada uno de los momentos de la investigación se va consolidando la población que se requiere, para dar sustento a cada momento. En este sentido Strauss \& Corbin (2002) especifican que se deben considerar cuatro aspectos relevantes en este muestréo teórico,

1. Se debe escoger un sitio o un grupo para ser estudiado. Esto, por supuesto, está guiado por la pregunta principal de la investigación. Por ejemplo, si un investigador está interesado en estudiar cómo toman decisiones los ejecutivos, debe ir a los lugares donde haya ejecutivos tomando decisiones para observar qué hacen y dicen verbalmente, por escrito, etc. También es importante obtener permiso de quien tenga la autoridad para acudir a estos sitios. 2. Se debe tomar una decisión sobre los tipos de datos que se van a usar. ¿Quiere el investigador usar observaciones, entrevistas, documentos, biografías, videos, casetes, o alguna combinación de los anteriores elementos? La decisión se debe tomar con base en aquellos datos que tienen el mayor potencial de captar los tipos de información deseados. Por ejemplo, un investigador puede querer emplear memorandos y documentos escritos, así como entrevistas y 
observaciones, cuando estudia la toma de decisiones de los ejecutivos. 3. Debe considerarse el tiempo que se dedicará al estudio de un área. Si un investigador está estudiando un proceso de desarrollo o un proceso evolutivo debe tomar decisiones iniciales acerca de seguir a determinadas personas o lugares, por un tiempo, o si debe seguir a diferentes personas o lugares en distintos tiempos. 4. Inicialmente, las decisiones con respecto al número de sitios y observaciones o entrevistas dependen del acceso, los recursos disponibles, los objetivos de la investigación y el tiempo y la energía del investigador. Más tarde, estas decisiones se pueden modificar a medida que vaya evolucionando la teoría. (pp. 222-223)

Estas recomendaciones nos dejan claro que no hay un volumen de muestra que sea el indicado, por el contrario, la pertinencia del muestreo depende de las características del contexto que se está evaluando, de las posibilidades de acceso a la información por parte del investigador y de la profundidad que a nivel teórico se genera en cada etapa del proceso. Para finalizar, es necesario aclarar que en el muestreo teórico se finaliza el proceso cuando las diferentes categorías se han saturado y en la recolección de la información no emergen nuevos datos relevantes al campo de conocimiento estudiado (Strauss \& Corbin, 2002).

\section{Educación e investigación.}

La anterior reflexión deja una propuesta a los educadores que piensan lo educativo, como una oportunidad para aportar a la transformación positiva de la sociedad y la cultura, ya que,

La educación en el siglo XXI se concibe como algo permanente y estructurado que permite al individuo tomar conciencia de sí mismo y de su entorno. Su misión es hacer madurar los talentos y capacidades de las personas, lo que impulsa al individuo a ser más autónomo y partícipe en la realización de su proyecto personal, ayudándole a desempeñar su función en la sociedad. (Sánchez, Sánchez, y Zaragoza, 2010, p. 497) 
Por lo tanto, esta perspectiva de la educación exige unos docentes actualizados académicamente, con competencias socio-emocionales que le permitan generar empatía hacia las realidades personales de los estudiantes, curiosos por la innovación en los aspectos pedagógicos y didácticos, con amplia preparación en su campo disciplinar, pero con la capacidad de estructurar colectivos de trabajo que relacionen las diferentes disciplinas considerando que

La educación no puede equiparar a cada estudiante con un conjunto completo de perspectivas; es más, estamos condenados al fracaso si intentamos hacer de cada joven un historiados, un biólogo, o un compositor de música clásica, Nuestro objetivo no debe ser compendiar la formación superior, sino facilitar a los estudiantes el acceso al corazón intelectual o alma profesional de las disciplinas. Dicho de otro modo: para tener éxito, la educación debe transmitir a los estudiantes una noción de las visiones del mundo que ofrecen las distintas disciplinas. (Gardner, 1999, p. 157)

Lo cual, solo es posible, si se consideran la aulas de clase como escenarios para la investigación en colectivo, para la transformación social y para la comprensión de las realidades culturales, lo cual implica desarrollar unas bases desde lo epistémico, desde lo organizativo, desde el aspecto metodológico y desde la formación de educadores en las distintas facultades de formación de docentes, en las escuelas, en los colegios y las universidades como una posibilidad para dar respuesta a las demandas que nos exige la sociedad en estos momentos de grandes cambios.

\section{Conclusiones}

Las problemáticas escolares en los diferentes contextos requieren de un diagnostico por parte de las instituciones educativas en los diferentes niveles de formación, es notable que cada estudiante asiste al aula de clase con intereses y particularidades diferentes, por lo tanto, los escenarios formativos deben responder a estas diferencias, pero es necesario identificarlas para hacer un abordaje correcto desde un ámbito educativo. Al respecto (Armstrong, 2012) 
especifica que es necesario celebrar las diferencias en las personas para encontrar en ellas los verdaderos aprendizajes.

Las continuas transformaciones en nuestra sociedad en los diferentes ámbitos implican también una visión distinta del educador, ya que "el profesor es la pieza clave de todo el sistema educativo. El nuevo papel de la educación y el conocimiento de la sociedad supone redefinir también el papel de los educadores" (Belmonte, 2003, p. 18). En este sentido, se debe redefinir el rol del educador en los planteles educativos, se debe invertir en una formación continua pero situada, se deben potenciar las competencias socio-emocionales en los docentes y la investigación en las aulas de clase debe ser parte de los pensum universitarios, de las actualizaciones pedagógicas y de las realidades de las instituciones en cada nivel formativo.

Así mismo, los procesos investigativos se deben involucrar como contenido en los entornos escolares, la indagación "in situ" como una posibilidad para comprender las realidades existentes, ya que "La investigación cualitativa pretende acercarse al mundo de "ahí fuera" (no en entornos de investigación especializada como los laboratorios) y entender, describir y algunas veces explicar fenómenos sociales "desde el interior" de varias maneras diferentes (Barbour, 2013, p. 16). En este sentido, provee una opción a los jóvenes, a los docentes y a la comunidad educativa para identificar las falencias, los avances y las perspectivas que se tienen en su realidad particular.

El interaccionismo simbólico es una apuesta epistemológica desde la sociología que permite al educador identificar las percepciones de los estudiantes a través de sus interacciones en el contexto en el que se desenvuelven. Ya que como lo afirma Piaget, (1961) "en la representación cognitiva hay, pues, adaptación al significado (es decir equilibrio entre la acomodación y la asimilación); en tanto que el significante consiste en imágenes reales o mentales, exactamente acomodadas o imitadas" (p. 225). Estas posibilidades de extraer las individualidades en lo investigativo le abren múltiples opciones al interaccionismo de conocer a profundidad la influencia del otro en el crecimiento individual. La teoría fundamentada como diseño metodológico le permite al educador sistematizar sus experiencias en sus 
diferentes manifestaciones. Permite categorizar los aspectos relevantes de la vivencia escolar, desde una producción teórica que se basa en los datos, en los apoyos teóricos, en la experticia del investigador y en la rigurosidad que provee el interaccionismo simbólico.

La educación a nivel mundial está presentando diferentes cambios en su forma, fondo y estructura, cada componente del acto educativo tiene que aportar en la adaptación a las nuevas realidades, "la experiencia nos ha enseñado que el ritmo de nuestros aprendizajes crece en calidad y cantidad cuando viene de la mano de buenos y expertos maestros mediadores" (Belmonte, 2003, p. 40), es el momento de propiciar cambios que emergan desde la capacidad y profesionalismo de los docentes transformadores en sus contextos de trabajo.

\section{Referencias}

Armstrong, T. (2012). El poder de la neurodiversidad. Barcelona-España: Paidós.

Barbour, R. (2013). Los grupos de discusión en investigación cualitativa. Madrid: Morata.

Belmonte, L. T. (2003). El perfil del profesor mediador. Madrid: Santillana.

Blumer, H. (1982). El interaccionismo simbólico: perspectiva y método. Barcelona: HORA, S.A.

Coffey, A., \& Atkinson, P. (1996). Making sense of cualitative data: complementary research strategies. United states: Sages publications.

Esteban, M. P. (2003). Investigación cualitativa en educación: fundamentos y tradiciones. Barcelona-España: McGraw-Hill/ interamericana de España, S. A. U.

Flick, U. (2012). Introducción a la investigación cualitativa. España: Morata. 
Gardner, H. (1999). The disciplined mind: beyond facts and standardized test, the K-12 education that every child deserves. New York: Penguin books.

Gardner, H. (2011). Verdad, belleza y bondad reformuladas: la enseñanza de las virtudes en el siglo XXI. Barcelona-España: Paidós.

Gardner, H., \& Davis, K. (2014). La generación APP. Barcelona- España: Paidós.

Hibbs, G. (2012). El análisis de datos cualitativos en investigación cualitativa. Madrid: Ediciones Morata.

Mounier, E. (1974). Obras tomo 1: 1931-1939. Barcelona: Laia.

Piaget, J. (1961). La formación del símbolo en el niño: imitación, juego y sueño. México: Fondo de cultura económica.

Piaget, J. (1999). Psicología de la inteligencia. Buenos Aires: Editorial Psique.

Ramírez, T. (2010). Cómo hacer un proyecto de investigación. Caracas- Venezuela: Corporación marca.

Sánchez, Y. C., Sánchez, A., \& Zaragoza, M. R. (2010). La evaluación y la información de calidad en educación física: propuesta de una herramienta de diagnóstico para la Educación Secundaria Obligatoria. Revista española de pedagogía, 497-520.

Strauss, A., \& Corbin, J. (2002). Bases de la investigación cualitativa. Técnicas y procedimientos para desarrollar la teoría fundamentada. Antioquia- Colombia: Imprenta Universidad de Antioquia.

Zuluaga, O. L. (2003). Epistemología de la pedagogía. Bogotá-Colombia: Cooperativa Editorial Magisterio. 